\title{
Ethnoscience et autres ethno « machins » aujourd'hui
}

Commentaires à propos du texte de Peter D. Dwyer

\section{Claudine Friedberg}

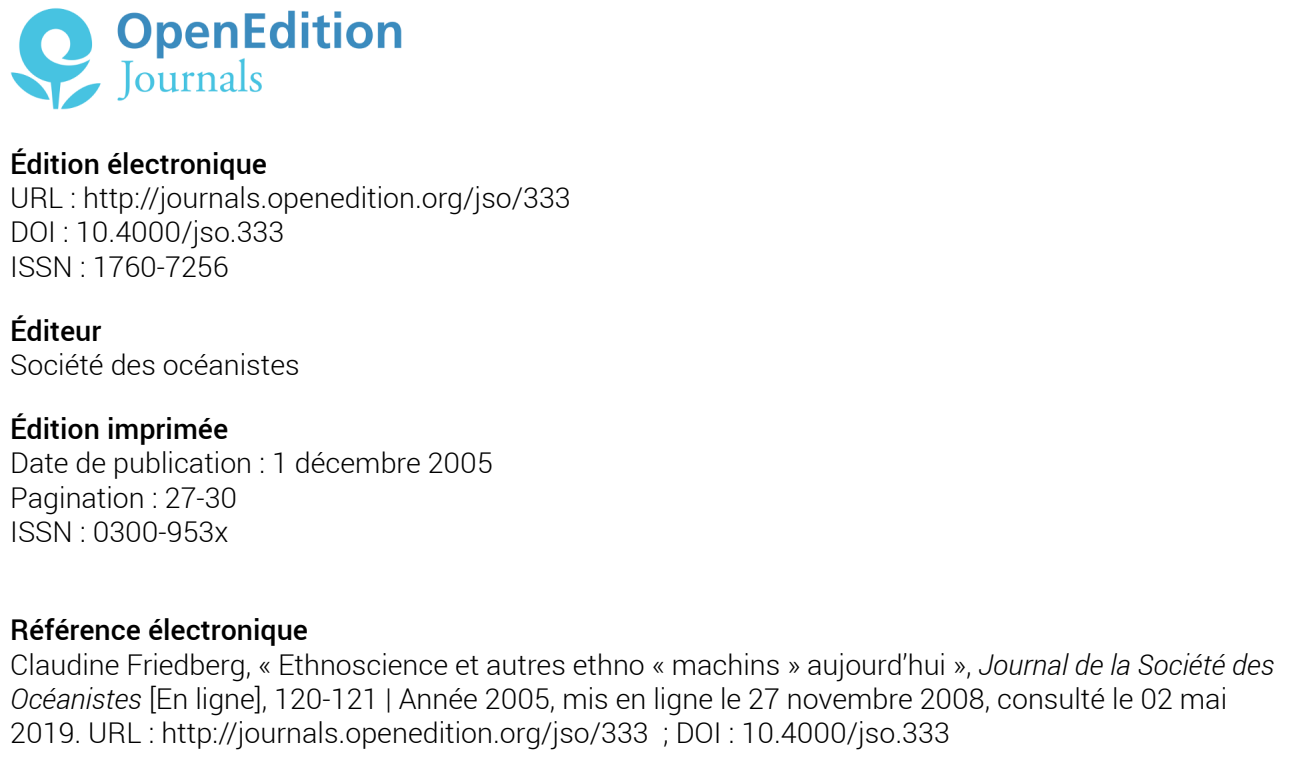

(c) Tous droits réservés 


\title{
Ethnoscience et autres ethno « machins » aujourd'hui. Commentaires à propos du texte de Peter D. Dwyer
}

par

\author{
Claudine FRIEDBERG*
}

Le grand mérite du texte de Peter Dwyer est de remettre en perspective les deux trajectoires des recherches sur les classifications populaires: l'une consacrée aux aspects cognitifs particulièrement incarnés par Brent Berlin, l'autre qu'il qualifie d'ethnoécologie magnifiquement représenté, pour ce qui est de l'aire océanienne, par Ralph Bulmer. Chacune de ces deux trajectoires se trouve maintenant incorporée dans deux types de problématique, celui de la psychologie évolutive pour la première, comme le remarque lui-même Peter Dwyer, et, pour la deuxième, celui de la gestion de l'environnement et de la biodiversité qui s'inscrit dans ce qu'il est maintenant convenu de désigner par l'expression «nouvelle gouvernance». Examinons ces deux aspects tout en montrant la difficulté de les séparer.

Pour ce qui est du premier, admettons qu'à une époque où l'on a progressé considérablement sur notre connaissance du fonctionnement mental des animaux et où l'on a découvert des capacités classificatoire chez les pigeons, il serait ridicule de mener des études sur les classifications populaires pour prouver, comme certains chercheurs en avaient encore l'intention dans les années 1970, que partout, dans toutes les sociétés, même les plus frustes sur le plan technique, les hommes sont capables de classer les plantes et les animaux de leur environnement. Plus personne aujourd'hui n'oserait douter de cette capacité. Cependant, la question demeure de savoir comment cette dernière s'exprime chez les humains.

Écartant les hypothèses modularistes ou essentialistes, controversées chez les spécialistes des neurosciences et qui relèvent plus de la perception que de la classification, penchons-nous sur la structure de ces classifications populaires et, en particulier, sur le problème de la hiérarchisation des catégories mises en évidence, question qui a beaucoup préoccupé les chercheurs, même s'il ne s'agit en aucune façon de vouloir retrouver une hiérarchisation comparable à celle de la systématique scientifique. Comme nous allons le constater, nous allons ainsi glisser progressivement dans les problématiques du deuxième type, c'est-à-dire s'inscrivant dans l'ethnoécologie.

Dès les travaux de Harold C. Conklin, il est admis, comme le rappelle Florence Brunois (supra, p. ?), que : " les ségrégats indigènes peuvent appartenir à plusieurs hiérarchies distinctes ». Chacune de ces hiérarchies correspond à un certain type d'usage ou à un point de vue déterminé. Par exemple, pour ce qui est des végétaux, ces hiérarchies peuvent être fondées sur des types d'usage médicinaux ou sur des critères de solidité pour des arbres utilisés dans la construction des habitations, ou encore sur une distinction entre plantes alimentaires sauvages et plantes cultivées. Il s'agit là de classifications relevant d'un objectif spécialisé par opposition aux clas-

* Département Hommes, natures, sociétés, MNHN, friedbg@mnhn.fr. 
sifications qui intéressaient les chercheurs voulant mettre en évidence des universaux, c'est-àdire des catégories indépendantes du contexte ; c'est ce que l'on a appelé « special purpose » par opposition à " general purpose » (Dwyer, infra, p. 3).

Dans tous les cas de figure, que l'on s'intéresse à l'aspect cognitif de la capacité classificatoire ou à la façon dont cette capacité s'inscrit dans un contexte particulier, la question des rapports hiérarchiques entre les catégories ne peut être éludée. Quel que soit l'appellation ou le statut qu'on lui donne, « taxon générique populaire » comme Brent Berlin ou "specième » comme Ralph Bulmer, il est un niveau qui joue un rôle stratégique sur le plan cognitif comme l'ont montré les expériences des psychologues (Rosch, 1976). C'est le " niveau de base », appelé aussi niveau zéro par certains chercheurs. Il correspond au «terme de base» dans la nomenclature appliquée aux objets naturels. Ces " termes de base » peuvent être simples, comme chêne ou noisetier, et n'ont alors pas d'autres sens dans la langue, ou composé comme bouton d'or ou reine des prés ; ce sont alors des appellations descriptives ou métaphoriques.

Les niveaux inférieurs sont alors désignés par un terme de base auquel on ajoute un ou plusieurs déterminants (par exemple chêne + vert, chêne + liège). Tous ces éléments sont dans des rapports d'inclusions hiérarchiques et ont tous au moins un caractère en commun. En restant dans le domaine végétal, on peut voir ainsi que chez les Bunaq (population du centre de Timor parlant une langue appartenant au phylum trans-néo-guinéen), toutes les plantes portant le terme de base balo ont en commun le même aspect, elles n'ont pas de tige et ont de grandes feuilles partant d'une base appelée wo. Il s'agit, en particulier, de plusieurs cultivars de taros (Colocasia esculenta) distingués par des déterminant différents, mais aussi d'un Colocasia sauvage vivant dans l'eau et appelé balo sai katoq, c'est-à-dire «balo sortie de grenouille ». De la même façon, tous les banians (plusieurs espèces de Ficus), arbres à latex (susuqil) qui sont des arbres commençant leur vie comme épiphyte sur d'autres arbres qu'ils finissent par étrangler, sont tous appelés pur avec des déterminants différents (pur + belis $«$ blanc », pur + apa gepal $«$ oreille de buffle », etc.).

Cette façon de désigner les plantes se retrouve partout dans le monde. Par exemple, en français, plusieurs petites plantes épineuses appartenant à des genres ou même des famille botaniques divers, sont appelées chardons avec des déterminants différents (chardon béni, chardon Roland...) et il en est de même pour les variétés de plantes cultivées (carottes + rouge + longue + de Croissy, choux + de Bruxelles...) $)^{1}$.

Qu'en est-il maintenant des catégories qui sont au-dessus du niveau de base et qui incluent des plantes portant des termes de base différents ? Les chercheurs à la quête d'universaux indépendants du contexte n'ont retenu que les catégories correspondant à des formes du vivant (life form) : arbre, herbe, liane... Mais ces catégories, sauf quand elles jouent un rôle de classificateurs obligatoires dans la langue, n'ont souvent qu'un aspect opératoire limité dans la vie des populations concernées. Par contre, les catégories opérationnelles associent le plus souvent un aspect propre aux plantes (morphologique ou écologique) avec leur rôle dans la vie de la société. Ainsi, chez les Bunaq, on trouve une catégorie hotel susuqil "arbres à latex », qui englobe toutes les plantes ayant un latex, parmi lesquels les Ficus étrangleurs et d'autres qui ne le sont pas et dont la sève sert de glue pour faire des pièges à oiseaux. Mais cette catégorie n'inclut pas que des arbres, elle comprend aussi des plantes arbustives comme le Calotropis gigantea ou même herbacées comme de petites Euphorbes. Encore plus caractéristique de cette association de critères appartenant à des domaines différents, la catégorie hotel upan gutu « arbres avec des pétioles comme ceux des feuilles de palmier ou de bananier ", regroupant ces dernières plantes qui, en plus de leur particularité morphologique, ont un statut particulier dans leur mode d'utilisation : elles sont sous la protection des « gardiens des produits du sol».

Cependant, en dehors de catégories englobantes fondées en partie sur l'usage, il en existe qui sont particulièrement intéressantes pour comprendre l'ordre qu'une société établit dans son espace de vie ; il s'agit des catégories organisatrices de l'espace qui peuvent en même temps jouer le rôle de catégorie englobante pour certains végétaux. C'est le cas, par exemple chez les Bunaq de bula qui comprend un certain nombre d'herbacées portant des termes de base différents et caractéristiques des prairies d'altitude à Timor. Mais, en même temps, le terme bula s'applique à un espace dans lequel le bétail peut paître et circuler, par opposition au matas momen, les terres de culture interdites à ce même bétail. À ce titre, bula peut comporter des zones arborées. On retrouve cette même complexité dans le terme lande en français : il se définit par 
une flore particulière, un type d'usage et, souvent, un statut foncier. Dans les sociétés où l'on pratique l'agriculture sur brûlis, il est fréquent qu'un seul terme désigne les jardins abandonnés, quel que soit le stade de régénération de la végétation. Même si un tel espace possède une flore particulière reconnue comme telle par la population locale (c'est la végétation secondaire des botanistes), à laquelle s'ajoutent souvent des plantes qui y ont été mises avant l'abandon précisément pour spécifier son statut, ce qui le caractérise est le fait que ceux qui l'ont utilisé en premier conservent sur lui un droit d'usage. C'est le cas par exemple chez les populations de l'ouest de l'île de Timor, en particulier chez les Dawan d'Insana où le terme utilisé pour désigner un tel espace est semo (Waluyo, 2000). On peut qualifier de "complexes" ces catégories définies par des critères appartenant à des domaines différents.

$\mathrm{Si}$, au-dessous du niveau de base, les rapports hiérarchiques paraissent évidents, ils le sont beaucoup moins quand on prend en compte les niveaux supérieurs. En effet, une même plante peut appartenir à plusieurs catégories englobantes différentes ou se trouver dans des situations intermédiaires entre plusieurs d'entre elles qui se chevauchent l'une l'autre.

Il est alors intéressant de voir s'il existe des rapports de subordination entre les critères de regroupement. Par exemple, toujours chez les Bunaq, dans l'ensemble hotel susuqil évoqué ci-dessus, il n'y a pas de liane à latex ; ces dernières sont classées parmi les lianes, le caractère lianescent est donc ici considéré comme plus important que le fait d'avoir du latex. Par contre, le fait d'avoir une tige lianescente épineuse n'entraîne pas pour les ignames d'être placées dans la catégorie des lianes ou des plantes épineuses. Elles sont classées dans celle des plantes à tubercules comestibles intitulée balo dik « taro igname » qui apparaît en particulier dans les textes rituels ou mythiques. Par contre, même si elle porte comme terme de base balo, le Colocasia sauvage balo sai katoq qui n'a pas de tubercule comestible n'appartient pas à la catégorie des balo dik mais à celle des plantes qui vivent dans ou à proximité de l'eau (il gie).

Ainsi, on se trouve plus souvent face à un ordonnancement des éléments composant l'environnement d'une société qu'à une véritable volonté de les classer, ordonnancement qui prend en compte les caractéristiques physiques, biologiques et comportementales de ces éléments, mais aussi leur rôle dans la vie des membres de cette société. Le plus important est alors de repérer les relations que ces derniers établissent entre ces différents éléments, entre ceux-ci et eux-mêmes et en y incluant les êtres de l'au-delà que l'on y trouve. Parmi ces relations, certaines ne concernent que les êtres vivants et relèvent donc de ce que l'on peut appeler l'ethnoécologie.

Par exemple, les Alune de Seram savent bien repérer les plantes consommées par les animaux, comme Helminthostachys zeylanica mangé par une tortue d'eau douce ou un type d'igname sauvage dont le Phalanger est friant ; même si les Alune apprécient eux-mêmes cette igname, quand ils en trouvent en forêt, ils n'y touchent pas, surveillent sa croissance et quand ils voient en fouillant au pied que le tubercule est assez gros, ils surveillent ses alentours pour chasser les Phalanger qui vont y venir en troupe (Suharno, 1997). Ils repèrent aussi les plantes qui sont toxiques pour d'autres plantes ; par exemple, autour de l'Endospermum moluccanum rien ne peut pousser. Mais, cette approche "écologique » inclut aussi les êtres de l'au-delà. Par exemple, Endospermum moluccanum est considéré éloigner les mauvais esprits, tandis qu'au contraire Octomeles sumatrana est considéré comme leur habitat.

L'ordonnancement de l'environnement des êtres qui vivent sur un territoire passe aussi par un ordonnancement de l'espace et la présence de certaines espèces servent à se repérer.

Comme le dit Peter Dwyer, on doit supposer que les capacités mentales que l'être humain a héritées de ses ancêtres étaient déjà le résultat d'une évolution qui s'était effectuée au sein de relations étroites avec les autres êtres vivants de son environnement dont sa survie dépendait. Il ajoute que le changement avec l'homme est qu'il ne se contente pas de percevoir et de concevoir cet environnement, mais qu'en plus il l'imagine. Il est évident que, pour l'homme, l'imagination joue un grand rôle dans sa façon de concevoir ses relations au monde. Mais, pour comprendre ces dernières, cette constatation ne suffit pas, il faut y ajouter l'émotion et l'affectivité.

Le relationnel est devenu la pierre de touche de toutes les recherches actuelles, quel que soit le domaine. Mais quand il s'agit des autres êtres vivants auxquels notre imagination prête des sentiments, l'affectif entre en jeu et, avec lui, l'émotion. Tout anthropologue ayant travaillé sur le terrain a pu le constater maintes fois, en particulier dans le déroulement des rituels au cours desquels le langage symbolique et métaphorique est déjà un premier effort de mise à distance. Ce sont tous ces aspects dont il faudrait tenir compte quand on parle d'associer les populations locales à la gestion de la biodiversité. 
Peter Dwyer a raison de dire que l'apparition d'un intérêt pour les TEK (traditional ecological kwnoledge) a désembourbé les recherches sur les classifications populaires de leur aspect purement cognitif. Malheureusement, ces connaissances écologiques locales sont souvent considérées sous leur seul angle technique et utilitaire sans prendre en compte les conceptions sociocosmiques dans lesquelles elles s'inscrivent, c'est-à-dire dans une mise en ordre de l'ensemble de l'univers.

L'analyse des catégories utilisées pour mettre de l'ordre dans l'environnement est une façon de mettre en évidence les pratiques techniques, mais aussi les conceptions que la population se fait de son action sur cet environnement, des relations qu'elle entretient avec les êtres qui le peuplent et $\mathrm{du}$ fonctionnement de l'ensemble du système dans lequel ils vivent tous ensemble, en y incluant les êtres de l'au-delà qui s'y manifestent, y compris les morts et les ancêtres.

Cependant cette analyse de la mise en ordre du monde doit être menée pour tous les acteurs intervenant dans la gestion d'un environnement donné. Ceci est particulièrement vrai dans les conflits qui surgissent entre les acteurs locaux eux-mêmes, et entre ces derniers et des gestionnaires venant de l'extérieur, aussi bien les représentants des administrations que les scientifiques. Chacun a en tête des justifications qui se réfèrent à un certain ordre du monde auquel il croit.

Si le souci de rentabilité économique immédiate est facile à déceler mais plus difficile à mettre en échec, la mise en évidence des idéologies, dont l'aspect le plus visible est l'universalisme et la coupure nature/culture, qui se dissimulent derrière les concepts des scientifiques naturalistes est rarement acceptée par ces derniers.

Comme nous le montre Peter Dwyer, les objectifs de l'ethnoclassification ont beaucoup évolué depuis son apparition au milieu du siècle dernier. C'est le chemin que nous a montré
Ralph Bulmer avec l'ethnoécologie qui s'est révélé le plus fructueux. Cependant, nous ne pouvons pas pour autant délaisser l'aspect cognitif des recherches sur les modes de catégorisation ; il est utile pour mieux appréhender les différents enjeux qui se dissimulent derrière la gestion de la biodiversité au niveau mondial et comprendre le rôle que les anthropologues doivent revendiquer dans ce domaine. $\mathrm{Ce}$ rôle, pour l'assumer, sans doute pourraient-ils le faire au nom de l'approche ethnologique en général, sans qu'il soit nécessaire de la saucissonner en différentes ethno "quelque chose ", ce qui embrouille plus que cela ne simplifie la démarche. En effet, une société et l'environnement réel et imaginaire, dans lequel elle vit, doivent être considérés comme un tout.

\section{RÉFÉRENCES BIBLIOGRAPHIQUES}

FrIEDBERG Claudine, 1990. Le savoir botanique des Bunaq. Percevoir et classer dans le Haut Lamaknen (Timor, Indonésie), mémoire du Muséum national d'histoire naturelle, tome 32 .

—, 1999. Diversity, order, unity. Different levels in folk knowledge about the living, Social Anthropology 7 , 1, pp. 1-16.

Rosch Éléonore, 1976. Classifications d'objets du monde réel : origine et représentations de la cognition, Bulletin de Psychologie, numéro spécial : La mémoire sémantique, S. Ehrlich, E. Tulving (éds), pp. 243-249.

SuHARno Myah Maria Wirawati, 1997. Représentation de l'environnement végétal et pratiques agricoles chez les Alune de Lumoli, Seram de l'ouest (Moluques centrales, Inonésie), thèse de doctorat de l'université Paris VI, spécialité : Écosystèmes forestiers tropicaux.

WaLuYo Eko Baroto, 2000. Les écosystèmes domestiqués par l'homme dans l'ancien royaume InsanaTimor, Reinwardtia 11, 5, pp. 295-417. 